\title{
Diagnóstico y prevención en pacientes con problemas de corazón
}

\author{
Diagnosis and prevention in patients with heart problems
}

\section{Diagnóstico e prevenção em pacientes com problemas cardíacos}

\author{
Miguel Á. Arteaga-Intriago I \\ miguel.arteag@gmail.com \\ María E. Oña-Rivas II \\ mariarivas@gmail.com \\ Saskia J. Mendoza-Garcia III \\ saskia.garcia@gmail.com
}

\author{
Luz P. Alcivar-Solórzano VI \\ luz.alcivar@gmail.com \\ Elio A. Santos-Andrade V \\ Elioa.santos@gmail.com \\ Abigail P. Saltos-Castro VI \\ abigailsaltos@gmail.com
}

Correspondencia: miguel.arteag@gmail.com

\footnotetext{
I. Médico General en funciones hospitalarias del Hospital de Especialidades, Portoviejo, Ecuador

II. Médico Residente área de emergencia Hospital IESS, Portoviejo, Ecuador.

III. Médico General Asistencial Hospital de Especialidades, Portoviejo, Ecuador.

IV Médico General Hospital Napoleón Dávila Córdova, Chone, Ecuador.

v Médico General en funciones hospitalarias Seguro Campesino, El Carmen, Ecuador.

VI Médico General Hospital General IESS, Manta, Ecuador.
} 


\section{Resumen}

Las enfermedades cardiovasculares constituyen una de las causas más importantes de discapacidad y muerte prematura en todo el mundo. El problema subyacente es la aterosclerosis, que progresa a lo largo de los anos, de modo que cuando aparecen los síntomas, generalmente a mediana edad, suele estar en una fase avanzada. Los episodios coronarios (infarto de miocardio) y cerebrovasculares (ataque apoplético) agudos se producen de forma repentina y conducen a menudo a la muerte antes de que pueda dispensarse la atención medica requerida. La modificación de los factores de riesgo puede reducir los episodios cardiovasculares y la muerte prematura tanto en las personas con enfermedad cardiovascular establecida como en aquellas con alto riesgo cardiovascular debido a uno o más factores de riesgo. Esta investigación tiene como objetivo general analizar el diagnostico y prevención de las personas con problemas cardiacos. La metodología empleada se baso en una investigación descriptiva, en documentación de campo y en la información descriptiva exploratoria. Los resultados obtenidos se basaron en un análisis de los tipos de enfermedades cardiacas, los mecanismos de diagnósticos utilizados, la prevención de los pacientes con enfermedad del corazón y la reducción de los riesgos por partes de los individuos. Como conclusión el diagnóstico y prevención va a depender de la enfermedad cardiaca que posea el paciente. Su recuperación y aumento de la calidad de vida es directamente proporcional al estilo de vida que desee llevar.

Palabras claves: Enfermedades del corazón, diagnostico, prevención, estilo de vida. 


\section{Abstract}

Cardiovascular diseases are one of the most important causes of disability and premature death worldwide. The underlying problem is atherosclerosis, which progresses over the years, so that when symptoms appear, usually at middle age, it is usually at an advanced stage. Acute coronary (myocardial infarction) and cerebrovascular (stroke) episodes occur suddenly and often lead to death before the required medical attention can be dispensed. The modification of risk factors can reduce cardiovascular events and premature death both in people with established cardiovascular disease and in those with high cardiovascular risk due to one or more risk factors. The general objective of this research is to analyze the diagnosis and prevention of people with heart problems. The methodology used was based on descriptive research, field documentation and descriptive exploratory information. The results obtained were based on an analysis of the types of heart diseases, the diagnostic mechanisms used, the prevention of patients with heart disease and the reduction of risks by individual parts. In conclusion, diagnosis and prevention will depend on the heart disease that the patient has. Your recovery and increase in quality of life is directly proportional to the lifestyle you want to lead.

Keys words: Heart disease, diagnosis, prevention, lifestyle. 


\section{Introducción.}

Unos de los grandes problemas de la sociedad son las enfermedades del corazón ya que son una de las primeras causas de muerte entre la población. Las enfermedades cardiovasculares constituyen actualmente una causa frecuente de invalidez y muerte prematura en el mundo (Armas Rojas, y otros, 2009).

El incremento de las tasas de mortalidad y la prevalencia de factores de riesgo (FR) observadas en Asia, África y América Latina, son los más importantes indicadores de la magnitud de la epidemia que está por venir (Ordúñez García, y otros, 2005). Lo cual estos continentes se diferencias de los países desarrollados debido a sus políticas de salud implementadas dentro de sus sociedades.

Ahora, se debe entender las enfermedades cardiovasculares partiendo del entendimiento sobre el funcionamiento del corazón. El corazón enferma porque agota la energía, degenera, se nutre mal o se infecta. Pierde la energía porque halla resistencia en la circulación periférica o lo intoxican venenos exógenos o endógenos (UAB, 2018).

Sus enfermedades repercuten sobre todo el organismo, mediante los trastornos de la circulación, que son de trascendencia suma en cuanto afectan al riñón, al pulmón, al bulbo, al hígado y, en general, a los órganos más importantes (UAB, 2018). Estas enfermedades cardiovasculares son problemas basados en el corazón y los vasos sanguíneos. Los ataques suelen ser fenómenos que se originan en obstrucciones que impiden a la sangre fluir hacia el corazón. La causa más frecuente es la formación de depósitos de grasa en las paredes de los vasos sanguíneos que irrigan este músculo (FUNICOR, 2012). 
Existen muchas cosas que pueden aumentar su riesgo de sufrir una enfermedad del corazón. Se les llama factores de riesgo. Algunos de ellos no se pueden controlar, pero hay muchos que sí. Aprender sobre ellos puede bajar su riesgo de tener una enfermedad cardíaca. Las enfermedades cardiovasculares más frecuentes son: cardiopatía coronaria, enfermedades cerebrovasculares, arteriopatías periféricas, cardiopatías congénitas, trombosis venosas profundas, embolias pulmonares y arritmias (Marly, 2018).

En el Ecuador las enfermedades del corazón están entre los primeros lugares de causa de muerte entre la población. En la Tabla 1 se puede observar las causas de muerte en la población de mujeres y hombres en el Ecuador para el 2008.

Existen diversas causas para catalizar los problemas cardiacos en el individuo. Uno de ellos es la obesidad. El sobrepeso y la obesidad se asocian a una mayor prevalencia de trastornos cardiovasculares, específicamente hipertensión arterial, hipercolesterolemia, diabetes y enfermedades del corazón. La asociación es más fuerte en los más jóvenes, y para la diabetes y la hipertensión arterial (Guallar-Castillón, y otros, 2002). Se previene y se trata con cambios en los estilos de vida, que incluyen dietas hipocalóricas e incremento de la actividad física (Soca \& Peña, 2009). 
Miguel Á. Arteaga-Intriago; María E. Oña-Rivas; Saskia J. Mendoza-Garcia; Luz P. Alcivar-Solórzano; Elio A. SantosAndrade; Abigail P. Saltos-Castro

Tabla 1. Causas de muerte en mujeres y hombres del Ecuador para el año 2008

\begin{tabular}{|c|c|c|c|}
\hline \multicolumn{4}{|l|}{ Mujeres } \\
\hline Enfermedad & Número & Taso* & $\%$ \\
\hline I. Dlabetes mellitus & | 931 & 28.0 & 7.6 \\
\hline 2. Enfermedades cerebro-vasculares & 1685 & 24.5 & 6.6 \\
\hline 3. Enfermedades hipertensivas & 1580 & 22.9 & 6.2 \\
\hline 4. Influenza y neumonia & | $52 \mid$ & 22.1 & 6.0 \\
\hline 5. Insuficlencla cardlaca & 1183 & 17.2 & 4.6 \\
\hline 6. Enfermedades Isquémicas del corazón & 1056 & 15.3 & 4.1 \\
\hline 7. Enfermedades del sistema urinario & 933 & 12.0 & 3.2 \\
\hline 8. Neoplasla maligna del estómago & 719 & 10.4 & 2.8 \\
\hline 9. Neoplasla maligna del útero & 708 & 10.3 & 2.8 \\
\hline I0. Cirrosis y otras enfermedades del higado & 665 & 9.7 & 2.6 \\
\hline \multicolumn{4}{|l|}{ Hombres } \\
\hline Enfermedad & Número & Tasa* & $\%$ \\
\hline I.Agresiones & 2279 & 32.9 & 6.6 \\
\hline 2.Accidentes de transporte terrestre & 2188 & 31.6 & 6.3 \\
\hline 3. Enfermedades cerebrovasculares & 1723 & 24.9 & 5.0 \\
\hline 4. Enfermedades isquémicas del corazón & 1704 & 24.6 & 4.9 \\
\hline 5. Enfermedades hipertensivas & 1685 & 24.4 & 4.9 \\
\hline 6. Influenza y neumonía & 1666 & 24.1 & 4.8 \\
\hline 7. Diabetes mellitus & 1579 & 22.8 & 4.6 \\
\hline 8. Insuficiencia cardiaca & 1134 & 16.4 & 3.3 \\
\hline 9. Cirrosis y otras enfermedades del higado & 1127 & 16.3 & 3.3 \\
\hline 10. Neoplasia maligna del estómago & 945 & 13.7 & 2.7 \\
\hline
\end{tabular}

Fuente: (Lucio, Villacrés, \& Henríquez, 2011)

Una causa habitual es la hipertensión arterial, que debe ser detectada y controlada a tiempo para prevenir y evitar el desarrollo de la insuficiencia cardiaca. La tensión arterial debe encontrarse por debajo de 140/90 mmHg en la consulta, y 135/85 si la medición la realiza el propio paciente o la familia en el domicilio. En el Ecuador para el año 2014 más de un tercio de la población mayor de 10 años (3 187 665) es prehipertensa y 717529 personas de 10 a 59 años padece de hipertensión arterial (De la Rosa \& Acosta Silva, 2017). También se puede relacionar la obesidad con la hipertensión arterial en la población ecuatoriana donde a mayor masa corporal mayor es la hipertensión arterial (Guato, 2012). 
También existen factores importantes que impulsan la causa de los problemas cardiacos y del cual se debe destacar es la depresión y ansiedad. Los trastornos causados por la depresión y la ansiedad pueden afectar al ritmo cardíaco, aumentar la presión arterial y alterar la coagulación de la sangre. También pueden elevar los niveles de insulina y colesterol (Valueoptions, 2018).

También el consumo excesivo de alcohol puede llevar a una situación de insuficiencia cardiaca, ya que daña el músculo cardiaco y origina la denominada miocardiopatía dilatada, una dilatación progresiva del corazón debido al adelgazamiento y debilitamiento de sus paredes.

Otros de los factores de riesgo se encuentran: Edad adulta, tabaquismo, sedentarismo, dislipidemia (elevación de colesterol y triglicéridos), herencia de enfermedad coronaria, consumo de cocaína y otros alucinógenos y diabetes (Marly, 2018).

Según Alianza Nacional para la Salud Hispana (2018) los síntomas varían dependiendo de cuanto se ha interrumpido el flujo de sangre que normalmente llega al órgano afectado. Cuando la interrupción del abastecimiento de sangre al cerebro o al corazón es severa, se puede sentir alguno o todos los siguientes síntomas:

Ataque al corazón:

$\checkmark \quad$ Dolor en el centro del pecho con una sensación de opresión o compresión que dura unos cuantos minutos.

$\checkmark \quad$ Dolor de pecho que se esparce al cuello, los hombros y / o a los brazos.

$\checkmark \quad$ Incomodidad en el pecho junto con ligereza en la cabeza, sudoración, desmayo, náuseas o respiración entrecortada. 
Derrame:

$\checkmark \quad$ Debilidad en los brazos o piernas.

$\checkmark \quad$ Pérdida de sensación en la cara o el cuerpo.

$\checkmark \quad$ Dificultad para hablar.

$\checkmark \quad$ Pérdida repentina de la visión en un ojo.

$\checkmark \quad$ Sentirse mareado o con dificultad para caminar.

$\checkmark \quad$ Dolor de cabeza repentino e intenso.

Insuficiencia cardiaca congestiva:

$\checkmark \quad$ Hinchazón de las extremidades inferiores llamada "edema periférico".

$\checkmark \quad$ Intolerancia al ejercicio seguido por respiración entrecortada, fatiga y tos.

(Hispana, 2018, p. 2-3)

Por lo tanto, esta investigación tiene como objetivo general analizar el diagnostico y prevención de los problemas del corazón. La metodología utilizada esta basada en una investigación descriptiva, documental de campo y exploratoria descriptiva tomando en consideración las características de las enfermedades del corazón, los tipos de problemas cardiacos y el diagnostico y prevención de los mismos.

\section{Método.}

La metodología utilizada para esta investigación se basó en los análisis sobre las enfermedades coronarias, las causas y síntomas de las mismas, a su vez se analizo los diferentes mecanismos utilizados para poder obtener un diagnostico eficaz para cada tipo de enfermedad 
cardiaca y por último poder determinar cuales son los factores de prevención para un paciente que sufre de alguna enfermedad del corazón. Esto se basó realizando una investigación descriptiva, en documentación de campo y en la información descriptiva exploratoria tomando en cuanto ciertos medios como lo son los sitios web, libros, revistas y entrevistas a personas que han padecido esta enfermedad

\section{Resultados.}

\section{Tipos de enfermedades del corazón}

Existen diversos tipos de enfermedades cardiovasculares. Los trastornos cardiovasculares se clasifican en cuatro tipos: enfermedades isquémicas del corazón, cerebrovasculares, vasculares periféricas y otras (Corella \& Ordovás, 2007). Esto se puede observar en la Figura 1.

Las enfermedades cerebrovasculares son enfermedades de los vasos sanguíneas que llevan la sangre al cerebro (Marly, 2018). Hay dos tipos. En las isquémicas se produce una disminución del flujo sanguíneo que llega a alguna región del cerebro, lo que produce necrosis tisular por daño neuronal irreversible. En las hemorrágicas, existe una extravasación de sangre por rotura de algún vaso (Corella \& Ordovás, 2007). En la Figura 2 se muestra la enfermedad cerebrovascular isquémicas y las hemorrágicas. 
Miguel Á. Arteaga-Intriago; María E. Oña-Rivas; Saskia J. Mendoza-Garcia; Luz P. Alcivar-Solórzano; Elio A. SantosAndrade; Abigail P. Saltos-Castro

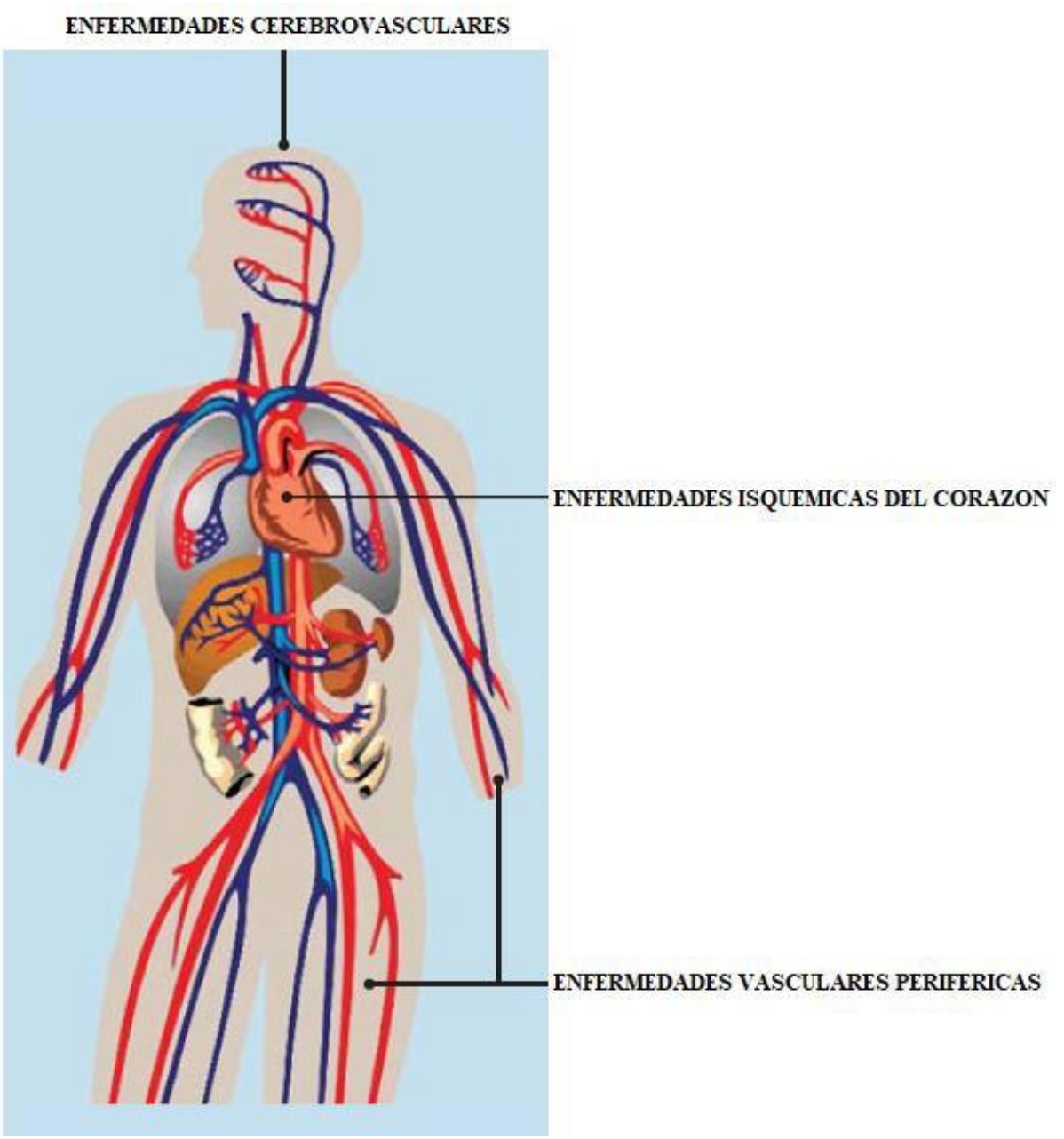

Figura 1. Tipos de enfermedades cardiovasculares. Fuente: (Corella \& Ordovás, 2007)

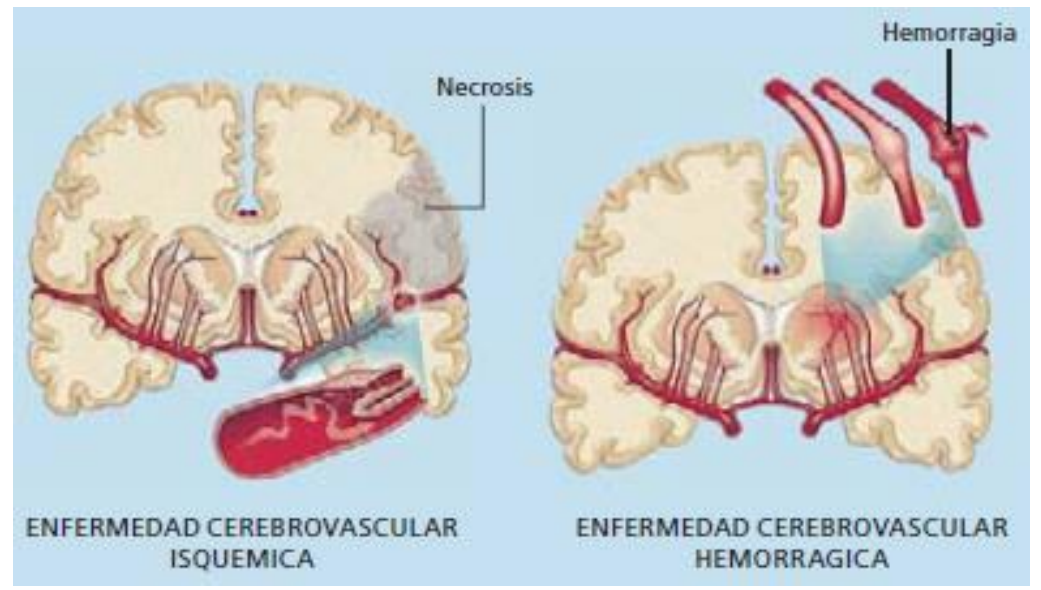

Figura 2. Los tipos de enfermedades cardiovasculares. Fuente: (Corella \& Ordovás, 2007) 
Las enfermedades de isquémicas del corazón se deben a un estrechamiento progresivo de la luz de las arterias coronarias, causado por la formación de la placa de ateroma (Corella \& Ordovás, 2007).

Es importante resaltar que dentro de las enfermedades isquémicas la obstrucción de las arterias es causada por el colesterol, células inflamatorias y productos de desecho. La obstrucción total de la arteria provoca la interrupción de la circulación de la sangre o isquemia; si este estado se prolonga, se destruye el tejido cardiaco, que da lugar a la zona de necrosis o infarto (Corella \& Ordovás, 2007). En la Figura 3 se detalla este tipo de enfermedad.

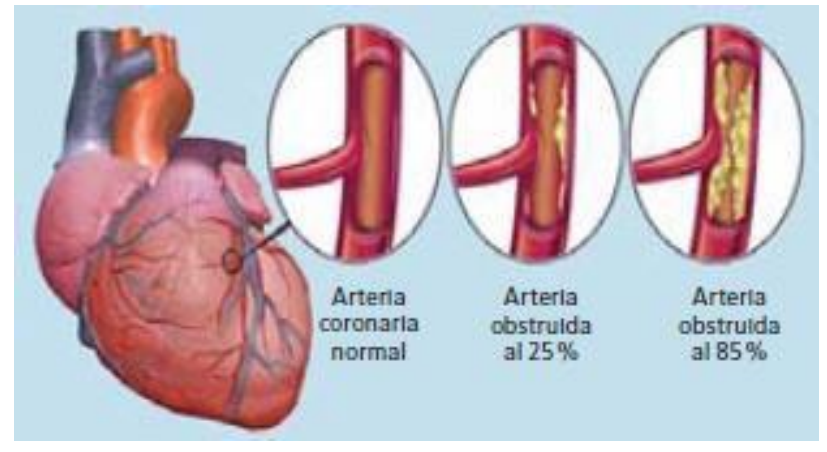

Figura 3. Detalle de obstrucción de las arterias del corazón.

Fuente: (Corella \& Ordovás, 2007)

Las enfermedades vasculares periféricas son trastornos de la circulación en los vasos (arterias o venas) que irrigan las piernas y los brazos. Enlentecen el flujo sanguíneo y provocan estrechamiento de los vasos, hinchazón y dolor. Puede causar isquemia (Corella \& Ordovás, 2007).

En la figura 4 se observa como es la obstrucción de las venas por coágulos de sangre, o trombos, que provocan oclusión y dan lugar a trombosis venosa. Si ese trombo se desprende, puede 
transportarse a los vasos de los pulmones y causar defunción por embolia pulmonar (Corella \& Ordovás, 2007).

Existen otros tipos de enfermedades del corazón las cuales se pueden describir a continuación:

Cardiopatía coronaria la cual es una enfermedad de los vasos sanguíneos que llevan la sangre al musculo cardiaco (corazón), arritmias son trastornos del ritmo cardiaco que involucran tanto los ritmos rápidos del corazón o taquicardias como los ritmos lentos denominados bradicardias y las cardiopatías congénitas que son malformaciones del corazón desde el nacimiento (Marly, 2018, p. 2).

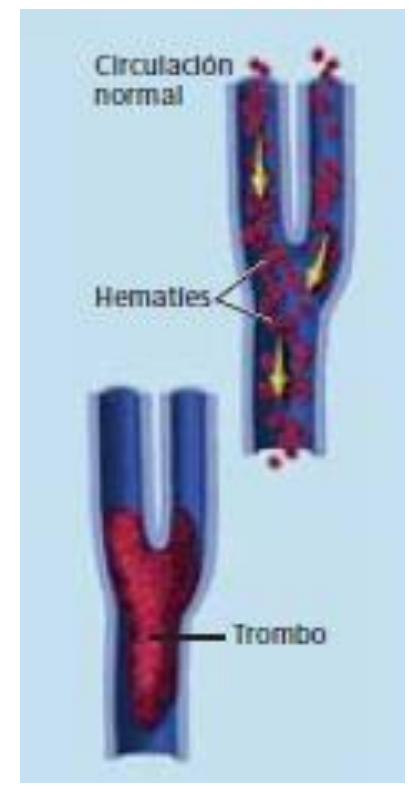

Figura 4. Formación de coágulos de sangre que pueden originar trombosis venosa.

Fuente: (Corella \& Ordovás, 2007) 


\section{Tipos de diagnósticos para detectar los problemas cardiacos}

Existen muchos mecanismos de diagnóstico para poder determinar o detectar los problemas cardiacos. Los electrocardiogramas registran señales eléctricas y puede ayudarle al médico a detectar irregularidades en el ritmo y la estructura del corazón. Se imprime un papel mediante una representación gráfica o trazado, donde se observan diferentes ondas que representan los estímulos eléctricos de las aurículas y los ventrículos (Marly, 2018).

También está el ecocardiograma que es una prueba no invasiva, que consiste en una ecografía del tórax, muestra imágenes detalladas de la estructura y el funcionamiento del corazón. Reconoce el tamaño y la forma de cada una de las cavidades y de los grandes vasos con el fin de identificar la extensión del daño causado por los infartos (Marly, 2018).

Otro es la prueba de esfuerzo implica aumentar la frecuencia cardíaca con ejercicios o medicamentos mientras se realizan estudios cardíacos y pruebas de diagnóstico por imágenes para controlar cómo responde el corazón. Con este examen además de evaluar la capacidad física del individuo y del comportamiento de la frecuencia cardiaca y de la presión arterial puede hacer el diagnostico de isquemia del corazón lo que implica riesgo de infarto al miocardio (Marly, 2018).

Además, esta la prueba de Holter el cual consiste es un dispositivo portátil que se debe usar para registrar un electrocardiograma continuo, por lo general de 24 a 72 horas. Es útil para evaluar pacientes con palpitaciones o para determinar la severidad de arritmia en pacientes con cardiopatía dilatada o después de infarto, como también para saber si hay fibrilación auricular intermitente como causa de infartos cerebrales (Marly, 2018). 
Miguel Á. Arteaga-Intriago; María E. Oña-Rivas; Saskia J. Mendoza-Garcia; Luz P. Alcivar-Solórzano; Elio A. SantosAndrade; Abigail P. Saltos-Castro

También está el monitoreo de presión arterial la cual registra la presión arterial durante 24 horas con el fin de determinar posibles episodios de hipotensión, evaluar la severidad de hipertensión o la efectividad del tratamiento (Marly, 2018).

\section{Factores de riesgo de la enfermedad cardíaca inherentes al individuo}

Su edad: Su riesgo de enfermedades del corazón sube a medida que envejece. Los hombres de 45 años o más y las mujeres de 55 años o más tienen un mayor riesgo

Sexo: Algunos factores de riesgo pueden afectar de manera diferente a las mujeres comparados con los hombres. Por ejemplo, el estrógeno brinda a las mujeres cierta protección contra las enfermedades del corazón, pero la diabetes aumenta más el riesgo de enfermedades del corazón en las mujeres que en los hombres

Raza o etnia: Ciertos grupos tienen mayores riesgos que otros. Los afroamericanos son más propensos que los blancos a tener enfermedades del corazón, mientras que los hispanos son menos propensos a sufrirlas. Algunos grupos asiáticos, como los asiáticos del este, tienen tasas más bajas, pero los asiáticos del sur tienen tasas más altas

Historia familiar: Usted tiene un mayor riesgo si tiene un familiar cercano que tuvo una enfermedad cardíaca a una edad temprana

\section{Tratamientos para combatir los problemas cardiacos}

Dependiendo de los distintos tipos de enfermedades se deben realizar los tratamientos pertinentes. Existen varias alternativas de tratamiento durante el curso de un infarto del miocardio, 
pero la forma más efectiva de tratamiento es el cateterismo cardiaco urgente y posible angioplastia coronaria (Marly, 2018).

En otros casos más complejos donde la enfermedad compromete todas las arterias del árbol coronario, se optará por el tratamiento quirúrgico de la enfermedad coronaria mediante una revascularización quirúrgica con puentes vasculares a las arterias obstruidas (Marly, 2018). En general, el tratamiento para las enfermedades cardíacas a menudo comprende lo siguiente:

Cambios en el estilo de vida. Entre ellos, seguir una dieta con bajo contenido de grasa y bajo contenido de sodio, hacer por lo menos 30 minutos de ejercicio moderado la mayoría de los días de la semana, dejar de fumar y limitar el consumo de alcohol.

Medicamentos. Si los cambios en el estilo de vida no son suficientes, es posible que el médico te recete medicamentos para controlar la enfermedad cardíaca. El tipo de medicamentos dependerá del tipo de enfermedad cardíaca.

Procedimientos médicos o cirugía. Si los medicamentos no son suficientes, es posible que el médico te recomiende procedimientos específicos o una cirugía. El tipo de procedimiento dependerá del tipo de enfermedad cardíaca y del grado de daño al corazón. Dentro de los procedimientos médicos o de cirugía se pueden determinar los siguientes:

El cateterismo cardiaco es un procedimiento invasivo que se realiza bajo anestesia local en la cual se toma como vía de acceso una ratería (a nivel de la ingle o del brazo), consiste en la introducción de un catéter que se conduce hasta donde se originan las arterias que llevan la sangre al corazón (arterias coronarias), se inyecta por el catéter un medio de contraste el cual permite ver las arterias coronarias en su interior. El equipo (cineangiógrafo) permite visualizar el 
recorrido del medio de contraste y mostrar el sitio de obstrucción arterial; esta obstrucción es posible eliminarla mediante una angioplastia coronaria en la mayoría de los casos. La angioplastia coronaria permite la eliminación de la obstrucción arterial mediante la utilización de un catéter balón, que se lleva al sitio de la obstrucción bajo la guía del cineangiógrafo donde se procede a inflar el balón el cual desplaza los elementos causantes de la obstrucción como la placa de aterosclerosis formada por fragmentos de colesterol, células inflamatorias y coágulos de sangre permitiendo la restauración del flujo de sangre en la arteria. La arteria tiende a volverse a cerrar al retirar el balón ya que sus paredes son elásticas. Para contrarrestar esta reacción de la arteria el médico implantará en el interior de la arteria afectada un stent. (Marly, 2018, p. 5)

En la Figura 5 se puede detallar el proceso de la enfermedad coronaria y el tratamiento con implante stent.

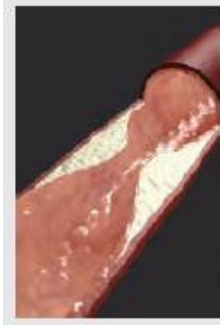

Arteria con enfermedad aterosclerótica

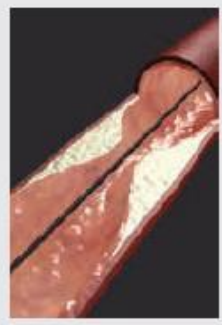

Arteria enferma con una guía de angioplástia superando la lesión

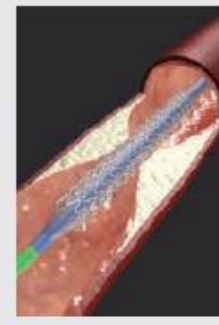

Estent coronario ubicándose en el sitio exacto de la lesión

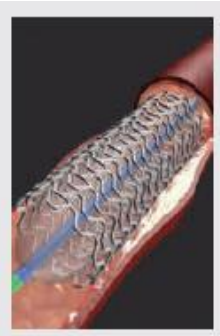

Implante estent coronario

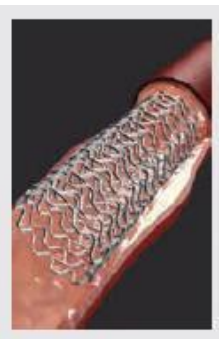

Estent coronario implantado y resultado final

Figura 5. Proceso de la enfermedad coronaria y el tratamiento con implante stent.

Fuente: (Marly, 2018)

Otra de las operaciones que se realizan para poder solventar la problemática de las arterias principales obstruidas es la realización del bypass coronario. Esta cirugía realiza un mecanismo que permite colocar un vaso sanguíneo como puente para sustituir a la arteria tapada y con ello poder 
distribuir la sangre al musculo cardiaco. Las arterias coronarias llevan sangre con oxígeno al músculo del corazón (miocardio). Esto es necesario para que el corazón pueda seguir latiendo continuamente y con la suficiente energía para bombear la cantidad de sangre necesaria en cada momento (FJD, 2018). Esta se puede observar en la Figura 6.

(A)

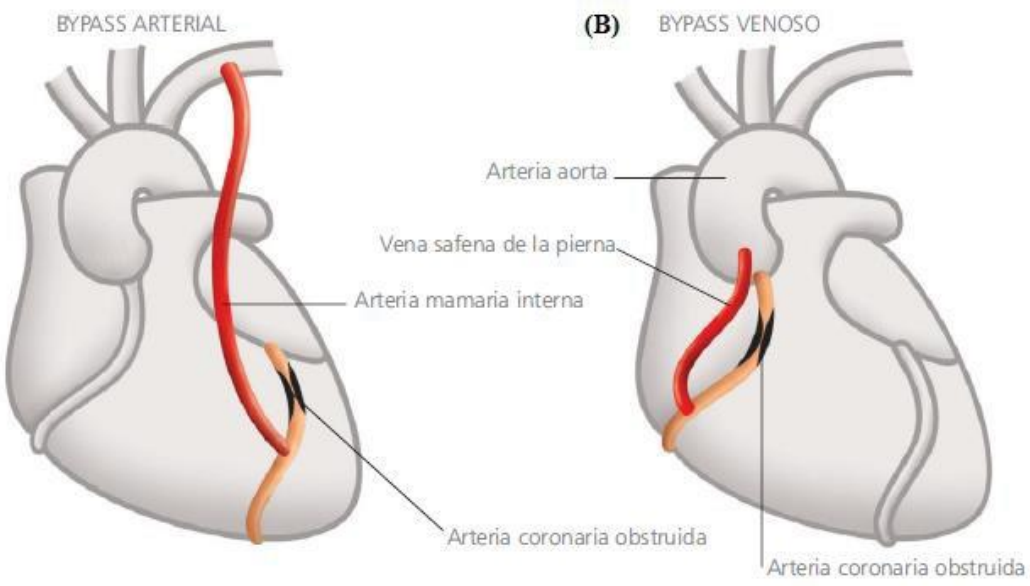

Figura 6. Tipos de bypass coronario. (A) Bypass Arterial y (B) Bypass Venoso.

Fuente: (FNR, 2018|)

\section{Prevención de los pacientes con problemas del corazón}

Para prevenir enfermedades cardiovasculares es necesario poder cumplir, por mérito propio, un cambio en el estilo de vida y con el cual aumenta la calidad de la misma. Según Alianza Nacional para la Salud Hispana (2018) adoptar comportamientos saludables puede reducir significativamente el riesgo de desarrollar condiciones cardiovasculares:

$\checkmark \quad$ Evite toda clase de uso de tabaco y humo de segunda mano.

$\checkmark \quad$ Empiece un programa regular de actividad física.

$\checkmark \quad$ Consuma alimentos saludables bajos en colesterol y grasas saturadas.

$\checkmark \quad$ Disminuya el consumo diario de sal y sodio. 
$\checkmark \quad$ Mantenga un peso saludable.

$\checkmark \quad$ Coma bastantes frutas y verduras. (p. 2)

Estos mismos factores de prevención sirven para la prevención de la hipertensión arterial. Los cambios de estilo de vida han demostrado efectividad en la disminución de la presión arterial. En aquellos casos que por sí solo no ha ocurrido, favorecen, pues disminuyen el número y dosificación de medicamentos hipotensores para lograr los niveles adecuados (Martínez, Pérez González, Córdoba Vargas, Santín Peña, \& Macías Castro, 1999).

Ahora, también existen tratamientos de prevención para la depresión debido a que esta afecta directamente al corazón. Los medicamentos antidepresivos prescritos, particularmente los inhibidores selectivos de recaptación de serotonina, son generalmente bien tolerados y seguros para los pacientes con afecciones cardíacas. Existen, sin embargo, posibles interacciones de ciertos medicamentos, así como efectos secundarios que requieren una vigilancia cuidadosa (Valueoptions, 2018).

Es importante resaltar la función del estado y de los entes no gubernamentales en los parámetros de prevención contra los problemas cardiacos. Estas políticas están basadas en: políticas destinadas a reducir el consumo de sal mediante normas en la fabricación de alimentos, políticas antitabaquismo y aumentar la disponibilidad de tratamiento médico para personas con enfermedades o condiciones asociadas a la enfermedad isquémica del corazón (Guzmán \& Ramírez, 2010).

\section{Reducción del riesgo de enfermedad cardíaca}

Afortunadamente, hay muchas cosas que usted puede hacer para reducir sus posibilidades de tener enfermedades del corazón: 
Controlar la presión arterial: La presión arterial alta (hipertensión) es un importante factor de riesgo para las enfermedades del corazón. Es importante que su presión arterial sea revisada regularmente, al menos una vez al año para la mayoría de los adultos, y más a menudo si tiene presión arterial alta. Tome medidas para prevenir o controlar la hipertensión, incluyendo cambios en su estilo de vida

Mantener los niveles de colesterol y triglicéridos bajo control: Los altos niveles de colesterol pueden obstruir sus arterias y aumentar su riesgo de enfermedad coronaria y ataque al corazón. Cambios en el estilo de vida y los medicamentos (si son necesarios) pueden bajar su colesterol. Los triglicéridos son otro tipo de grasa en la sangre. Los altos niveles de triglicéridos en la sangre también pueden aumentar el riesgo de enfermedad coronaria, especialmente en mujeres

Mantener un peso saludable: Tener sobrepeso u obesidad puede aumentar su riesgo de padecer enfermedades cardíacas. Esto es porque están vinculados a otros factores de riesgo de enfermedad del corazón, incluyendo elevados niveles de colesterol y triglicéridos, presión arterial alta y diabetes. Controlar su peso puede reducir estos riesgos

Alimentarse con una dieta saludable: Trate de limitar las grasas saturadas, los alimentos ricos en sodio y azúcares agregados. Coma mucha fruta fresca, verduras y granos enteros. La dieta DASH es un ejemplo de un plan de alimentación que puede ayudarle a bajar la presión arterial y el colesterol, lo que puede reducir el riesgo de enfermedades del corazón

Hacer ejercicio regularmente: El ejercicio tiene muchos beneficios, fortalece su corazón y mejora su circulación. También puede ayudarle a mantener un peso saludable y bajar el colesterol y la presión arterial. Todo esto puede reducir el riesgo de enfermedades del corazón 
Limitar el alcohol: Beber demasiado alcohol puede aumentar su presión arterial y añadir calorías adicionales, lo que puede causar aumento de peso. Ambos aumentan el riesgo de enfermedades del corazón. Los hombres no deben consumir más de dos bebidas alcohólicas al día, y las mujeres no deben tomar más de una

No fumar: Fumar cigarrillos eleva su presión arterial y lo pone en mayor riesgo de ataque cardíaco y accidente cerebrovascular. Si no fuma, no comience ahora. Si usted fuma, dejar de fumar disminuirá su riesgo de sufrir una enfermedad cardíaca. Usted puede hablar con su médico para que le ayude a encontrar la mejor manera de dejar de fumar

Controlar el estrés: El estrés está vinculado a las enfermedades del corazón de varias maneras. Puede aumentar la presión arterial. El estrés extremo puede ser un "desencadenante" de un ataque al corazón. Además, algunas maneras comunes de hacer frente al estrés, como comer en exceso, beber mucho y fumar, son dañinas para el corazón. Algunas formas de ayudar a controlar su estrés incluyen hacer ejercicio, escuchar música, enfocarse en algo tranquilo o sereno y meditar

Controlar la diabetes: Tener diabetes duplica su riesgo de enfermedad cardíaca diabética. Esto se debe a que, con el tiempo, el alto nivel de azúcar en la sangre por la diabetes puede dañar los vasos sanguíneos y los nervios que controlan el corazón y los vasos sanguíneos. Por lo tanto, es importante hacerse la prueba de la diabetes y, si la tiene, mantenerla bajo control

Asegurarse de dormir lo suficiente: Si no duerme lo suficiente, aumenta el riesgo de hipertensión, obesidad y diabetes. Estas tres condiciones pueden aumentar su riesgo de enfermedades del corazón. La mayoría de los adultos necesitan de siete a nueve horas de sueño por noche. Asegúrese de tener buenos hábitos de sueño. Si tiene problemas frecuentes para dormir, 
hable con su proveedor de atención médica. La apnea del sueño, por ejemplo, hace que las personas dejen de respirar brevemente muchas veces durante el sueño. Esto interfiere con su capacidad de obtener un buen descanso y puede aumentar su riesgo de enfermedad cardíaca. Si cree que podría tenerla, pregúntele a su médico si debería realizarse un estudio del sueño. Y si tiene apnea del sueño, asegúrese de recibir tratamiento

\section{Conclusiones.}

Unos de los principales organismos del ser humano es el corazón porque se desempeña como un motor que impulsa las fuerzas físicas y psicológicas necesarias para los individuos. El funcionamiento del corazón va a depender del estilo de vida que tenga la persona. Si hay aumento de comidas no saludables, altas en colesterol y sales, como también el uso del tabaco o drogas, sin olvidar el sobrepeso, obesidad y sedentarismos. Todos estos factores impulsaran a un a enfermedad cardiaca y por lo cual disminuirá la calidad de vida del individuo. Las enfermedades cardiacas se pueden dividir en distintos grupos como las enfermedades cerebrovasculares, las enfermedades isquémicas del corazón, las enfermedades vasculares periféricas entre otras. Cada una de ellas generadas por unas causas y por ende manifestadas a través de unos síntomas que producirán un rápido diagnostico con el fin de determinar u tratamiento específico.

Las causas de estas enfermedades son producto de un estilo de vida descontrolado lo cual produce para unos casos pequeños coágulos de sangre en el cerebro, en otros produce obstrucción de las arterias por grasa y también produce coágulos de sangre en las venas de los miembros inferiores haciendo producir una trombosis venosa. Es por ello necesario poder tomar las indicaciones médicas y llevar una vida sana libre de todas las sustancias que pongan en riesgo la vida. Los síntomas que se 
producen son opresión en el pecho que puede irradiarse hacia el cuello, brazos y mandíbula, sensación de fatiga con pequeños esfuerzos, náuseas y sudoración profusa.

Poder determinar el diagnóstico es fundamental porque con ello conlleva a aplicar el tratamiento adecuado. Existen varios mecanismos de diagnostico como lo son el electrocardiograma, prueba de esfuerzo, prueba de holter, monitoreo de presión arterial entre otros. Cada uno de ellos puede verificar el daño causado por la enfermedad y poder registrar un diagnostico eficaz. Entre los tratamientos se encuentran los tratamientos quirúrgicos y los tratamientos endovenosos. Los tratamientos quirúrgicos han servido de ayuda para solventar los grandes problemas desde hace un largo tiempo, pero lamentablemente pueden generar efectos secundarios ya que es una cirugía invasiva. Uno de las principales intervenciones quirúrgicas es el bypass coronario. Los tratamientos endovenosos son menos invasivos y producen una mejora calidad de vida solo con la salvedad que son más costosos por la alta tecnología utilizada. Unos de los procesos utilizados es aplicación de microstent vía venosa hacia la arteria o vena obstruida.

Por lo tanto, el diagnostico y prevención va a depender de la enfermadad cardiaca que posea el paciente. Su recuperación y aumento de la calidad de vida es directamente proporcional al estilo de vida que desee llevar.

\section{Referencias}

Armas Rojas, N. B., Dueñas Herrera, A., de la Noval García, R., Castillo Guzmán, A., Suárez Medina, R., Varona Perez, P., \& Bonet Gorvea, M. (2009). Enfermedades del corazón y sus características epidemiológicas en la población cubana de 15 años y más. Revista Cubana de Investigaciones Biomédicas, 28(4), 1-13.

Corella, D., \& Ordovás, J. M. (2007). GENES, DIETA Y ENFERMEDADES CARDIOVASCULARES. INVESTIGACION Y CIENCIA, 74-83. 
De la Rosa, J. M., \& Acosta Silva, M. (2017). Posibles factores de riesgo cardiovasculares en pacientes con hipertensión arterial en tres barrios de Esmeraldas, Ecuador. Revista Archivo Médico de Camagüey, 21(3), 361-369.

FJD. (08 de Diciembre de 2018). Cirugia de revascularización coronaria. Obtenido de https://www.fjd.es/.../21686-Cirugia\%20de\%20revascularizacion\%20coronaria.pdf

FNR. (08 de Diciembre de 2018|). ¿Que es el bypass coronario? Obtenido de http://www.fnr.gub.uy/sites/default/files/info_pacientes/folleto_bypass.pdf

FUNICOR. (2012). A cuidar el corazón. Fundación Internacional del Corazón.

Guallar-Castillón, P., Banegas, J. R., Yébenes, M. J., Gutiérrez-Fisac, J. L., García, E. L., \& Rodríguez-Artalejo, F. (2002). Asociación de la enfermedad cardiovascular con el sobrepeso y la obesidad en España. Medicina clínica, 118(16), 616-618.

Guato, E. (2012). Grados de hipertensión arterial en relación con sobrepeso y obesidad, en los pacientes que acuden al servicio de consulta externa; de medicina interna y cardiología del hospital del IESS Ambato en el periodo septiembre 2010 a febrero 2011|. Ambato - Ecuador: Trabajo de Grado - UNIVERSIDAD TÉCNICA DE AMBATO.

Guzmán, R. G., \& Ramírez, J. A. (2010). Enfermedad isquémica del corazón, epidemiología y prevención. Revista de la Facultad de Medicina UNAM, 53(5), 35-43.

Hispana, A. N. (10 de Diciembre de 2018). ENFERMEDADES CARDIOVASCULARES. Obtenido de https://www-tc.pbs.org/americanfamily/pdf/cardio_esp.pdf

Lucio, R., Villacrés, N., \& Henríquez, R. (2011). Sistema de salud de Ecuador. Salud Pública de México, 53, 177-187.

Marly, C. d. (10 de Diciembre de 2018). Enfermedades cardiovasculares, sintomas y tratamientos. Obtenido de http://marly.com.co/wp-content/uploads/2018/10/FOLLETOENFERMEDADESCARDIOVASCULARES.

Martínez, C., Pérez González, R., Córdoba Vargas, L., Santín Peña, M., \& Macías Castro, I. (1999). Programa nacional de prevención, diagnóstico, evaluación y control de la hipertensión arterial. Rev Cubana Med Gen Integr, 15(1), 46-88.

Ordúñez García, P. O., Cooper, R. S., Espinosa Brito, A. D., Iraola Ferrer, M. D., Bernal Muñoz, J. L., \& La Rosa Linares, Y. (2005). Enfermedades cardiovasculares en Cuba: determinantes para una epidemia y desafíos para la prevención y control. Revista Cubana de Salud Pública, 31(4), 1-15.

Soca, P. E., \& Peña, A. N. (2009). Consecuencias de la obesidad. Revista Cubana de Información en Ciencias de la Salud (ACIMED), 20(4), 84-92. 
Miguel Á. Arteaga-Intriago; María E. Oña-Rivas; Saskia J. Mendoza-Garcia; Luz P. Alcivar-Solórzano; Elio A. SantosAndrade; Abigail P. Saltos-Castro

UAB. (10 de Diciembre de 2018). Enfermedades del Corazón. Obtenido de https://ddd.uab.cat/pub/llibres/1914-1930/60248/patterespanidom_a1914-1930t2f2r3x2.pdf

Valueoptions. (10 de Diciembre de 2018). La Depresión y las Enfermedades Cardíacas. Obtenido de http://www.valueoptions.com/spotlight_heart/html/pdfs/Articles/Spanish/Depression\%20Esp ano 